\title{
Modified Lisfranc Amputation to Reduce Hematoma Rate in Diabetic Foot Ulcer
}

\author{
Sooseong Leem, M. Seung Suk Choi, Jung Woo Chang, Jang Hyun Lee \\ Department of Plastic and Reconstructive Surgery, College of Medicine, Hanyang University, Seoul, Korea
}

\begin{abstract}
Background: Diabetic foot ulcers (DFU) may result in limb amputations. Lisfranc level amputations follow the tarsometatarsal joint line, which is indented at the second toe ray due to the shortness of the intermediate cuneiform bone. For this reason a dead space inevitably forms at this site underneath the skin. We describe a modified technique for amputations at Lisfranc level obliterating this dead space, which in turn may result in less hematoma.

Methods: Four patients with DFU were operated using a modified Lisfranc amputation. The amputations were performed along the tarsometatarsal joints except for the second toe ray. After enucleation of the first, third, fourth and fifth metatarsals with the scalpel, the second metatarsal was amputated with an oscillating saw at the level of the distal ends of the medial and lateral cuneiforms, leaving the proximal portion of the metatarsal bone connected to the tarsus, thus obliterating the dead space between the cuneiforms. The skin was closed primarily.

Results: Mean follow-up was 16.2 months. All patients were male and their mean age was 69 years. All stumps healedprimarily without developing a hematoma. One of the patients is able to walk on his salvaged foot without orthotic device. The other patientsare in the status of rehabilitation.

Conclusion: The described modification can efficiently reduce the dead space resulting from classic amputation at the Lisfranc level. It remains to be shown in a larger study, if a reduced hematoma rate also results in a lower overall complication rate.
\end{abstract}

Keywords: Lisfranc amputation, Diabetic foot, Midfoot amputation, Diabetic foot ulcer, Modified Lisfranc amputation

\section{Introduction}

The number of diabetes mellitus patient is an increasing [1]. Over 10\% of the population in Korea are affected [2]. And its complications are major cause of morbidity and mortality in Korea [3]. One of major complications of diabetic mellitus is diabetic foot ulcer. And diabetic foot ulcers are the most common reasons leading to foot amputation [4]. One of the options in amputating foot is Lisfranc amputation. Compared to the more traditional transmetatarsal amputation, Lisfranc amputation is performed at more proximal level, giving an alternative surgical option when a transmetatarsal amputation is not appropriate [5]. Lisfranc level amputations follow the tarsometatarsal joint line, which is indented at the second toe ray due to the shortness of the intermediate cuneiform bone (Fig. 1). For this reason a dead space inevitably forms at this site underneath the skin.

We describe a modified technique for amputation at Lisfranc level obliterating this dead space, which in turn may result in less hematoma and seroma.

\section{Methods}

The medical records of 4 patients who underwent out modified Lisfranc amputation
Original Article

Received: September 25, 2017

Revised: September 27, 2017

Accepted: September 27, 2017

\section{Corresponding author:}

M. Seung Suk Choi, M.D.

Department of Plastic and Reconstructive Surgery, College of Medicine, Hanyang University, 153 Gyeongchun-ro, Guri 11923,

Korea

Tel: +82-31-560-2330

Fax +82-31-560-2338

E-mail: msschoi1@gmail.com

No potential conflict of interest relevant to this article was reported.

This is an Open Access article distributed under the terms of the Creative Commons Attribution Non-Commercial License (http://creativecommons.org/licenses/by-nc/4.0/) which permits unrestricted non-commercial use, distribution, and reproduction in any medium, provided the original work is properly cited.

C 2017 Korean Wound Management Society 


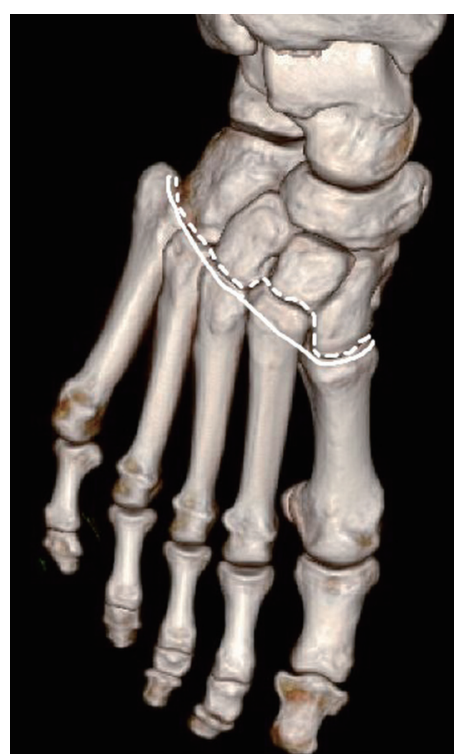

Fig. 1. The original Lisfranc line is indented at the 2nd tarsometacarpal joint (dotted line). This place is prone to hematoma formation after Lisfranc amputation. The modified Lisfranc amputation runs in a straight line from the 1st and the 3rd tarsometacarpal joints, and a bone plug in left in situ (solid line) obliterating the dead space.

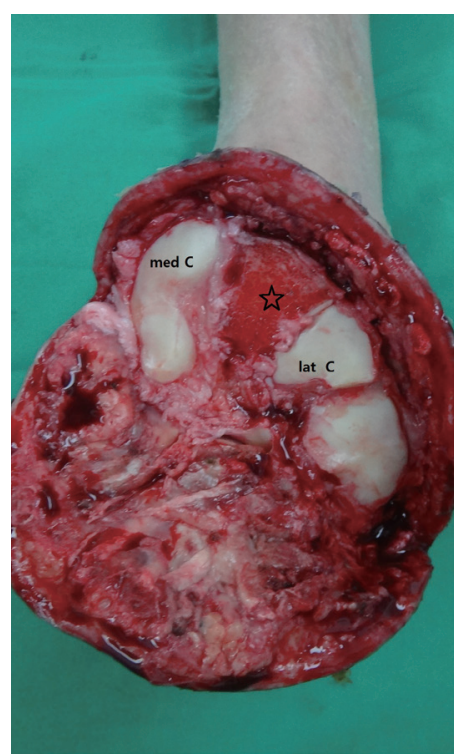

Fig. 2. Intraoperative photo of after modified Lisfranc amputation in a 70 years old male: The retained portion of the 2nd metatarsal base (asterisk) fills the space between the medial ("med C") and lateral cuneiform bones ("lat C").

Table 1. Patient information

\begin{tabular}{lcccccl}
\hline Patient No. & Sex/Age & Underlying disease & \multicolumn{1}{c}{ Amputation method } & F/U & \multicolumn{1}{c}{ Result } & Postoperative state \\
\hline 1 & M/70 & DM & Modified Lisfranc amputation & $34 \mathrm{~m}$ & No hematoma & Ambulation \\
2 & M/65 & DM/HTN/ESRD & Modified Lisfranc amputation & $32 \mathrm{~m}$ & No hematoma & Rehab \\
3 & M/74 & DM/HTN/MI & Modified Lisfranc amputation & $3 \mathrm{~m}$ & $\begin{array}{l}\text { No hematoma } \\
\text { Wound problem }\end{array}$ & Rehab \\
4 & M/67 & DM/Ml & $\begin{array}{l}\text { Modified Lisfranc amputation } \\
\text { (+ Achilles tendon lengthening) }\end{array}$ & $2 \mathrm{~m}$ & $\begin{array}{l}\text { No hematoma } \\
\text { Wound problem }\end{array}$ & Death d/t Ml \\
\hline
\end{tabular}

DM, diabetes mellitus; ESRD, end stage renal disease; MI, myocardial infarction transluminal angioplasty.

were reviewed. From July 2015 to August 2016, four patients with diabetic foot ulcers were operated using a modified Lisfranc amputation by a single surgeon (Fig. 1).

The patient was placed supine on the operation table. A thigh tourniquet was applied with $300-350 \mathrm{mmHg}$ pressure. The incision was designed to create long dorsal and plantar flaps. The amputations were performed along the tarsometatarsal joints except for the second toe ray. After enucleation of the first, third, fourth and fifth metatarsals with the scalpel, the second metatarsal was amputated with an oscillating saw at the level of the distal ends of the medial and lateral cuneiforms, leaving the proximal portion of the metatarsal bone connected to the tarsus, thus obliterating the dead space between the cuneiforms (Fig. 2). The skin flaps were trimmed in fish-mouth fashion to allow for primary closure. The skin was closed primarily after insertion of a drain. A splint was applied in all cases.

Seroma formation, hematoma, wound problem which needed revision, wound infection was recorded. Drain was removed 1-4 days after operation depending on amount of bleeding through the drain. Stitches were removed at approximately 21 days after operation depending on wound status.

\section{Results}

Total of 4 diabetic foot patients underwent Modified Lisfranc Amputation. Patient characteristics are shown in Table 1. All patients were male and their mean age was 69 years (range, 65-74 years). Mean follow-up was 16.2 months (range, 2-34 months). All stumps healed primarily without developing a hematoma or seroma (Fig. 3). One of the patients is able to walk on his salvaged foot without orthotic device (Fig. 4). The other patients are in the status of rehabilitation.

\section{Discussion}

The number of people with diabetes are continuously growing, and obviously the global prevalence of the diabetic foot 


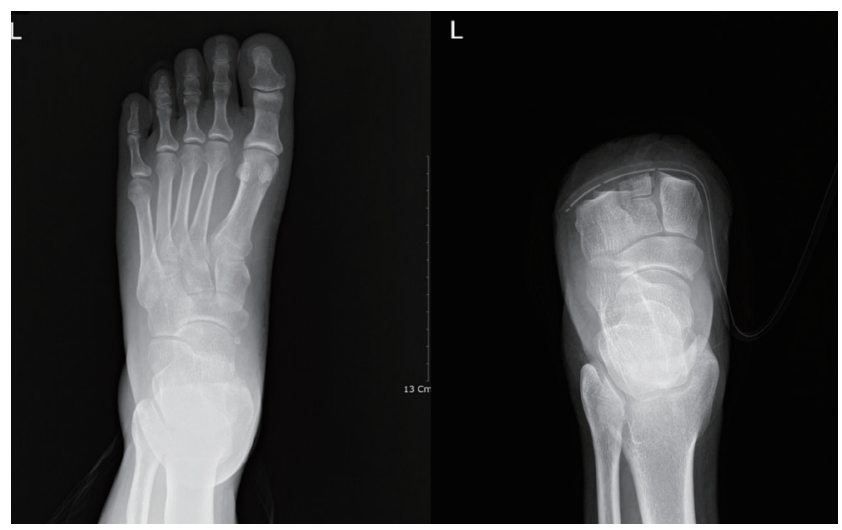

Fig. 3. Preoperative and postoperative X-ray of modified Lisfranc amputation: Note that there is no dead space remaining after surgery.

has risen [6]. The treatment of diabetic foot disease poses difficulties not only surgeon but patients, not only because of disease if self but patients suffer co-morbidities. One of the most common and serious complication is diabetic foot ulcers, as wound healing is impaired in the diabetic patient, than patient whom do not have underlying pathophysiological defect $[7,8]$. And also, in case of seroma or hematoma, tissue repair is impaired and the healing process is delayed which could lead to wound infection [9].

Dead space following surgical closure can lead to hematoma and seroma, which give and environment conducive to bacterial growth $[10,11]$. Inserting a drain will be helpful with these problems but leaving a drain for a long time is not possible because prolonged drainage increases the risk of infection [12]. Unlike another operation, in an operation like Lisfranc amputation, other method use to obliterate dead space, such as quilting suture, use of sealants and sclerotherapy or compression dressing, cannot be performed due to its anatomic structure and lack of circulation in diabetic foot. So obliterating the dead space anatomically, by leaving the proximal portion of the metatarsal bone, is a great idea and one of few options that can be made in Lisfranc amputation.

Wound problem that occurred in two cases were minor and healing with only with dressing and no additional surgical procedure, such as revision, were required. None of the patients had hematoma. Postoperative X-ray showed that there is no dead space in which fluid accumulation can be made (Fig. 3).

The described modification can efficiently reduce the dead space resulting from classic amputation at the Lisfranc level, resulting low hematoma or seroma formation. A larger study

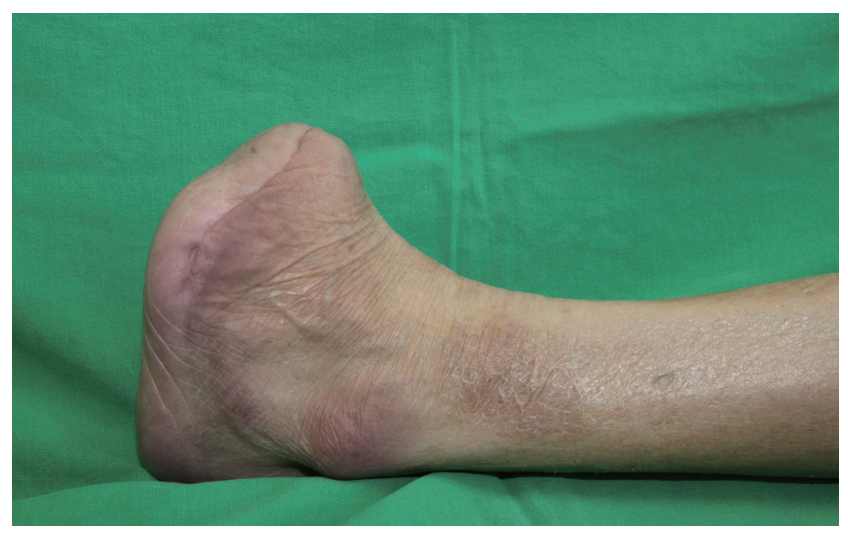

Fig. 4. Amputated stump 18 months after modified Lisfranc amputation.

should be done with comparison between classic Lisfranc amputation.

\section{References}

1. Wild S, Roglic G, Green A, et al. Global prevalence of diabetes: estimates for the year 2000 and projections for 2030. Diabetes Care 2004;27:1047-53.

2. Statistics Korea: Prevalence of diabetes mellitus. [updated 2016 Dec 27]. Available from: http://www.index.go.kr.

3. Kim DJ. The epidemiology of diabetes in Korea. Diabetes Metab J 2011;35:303-8.

4. Armstrong DG, Lavery LA. Diabetic foot ulcers: prevention, diagnosis and classification. American Family Physician 1998; 57:1325-8.

5. Greene CJ, Bibbo C. The Lisfranc Amputation: A More Reliable Level of Amputation With Proper Intraoperative Tendon Balancing. J Foot Ankle Surg 2017;56:824-826. doi: 10.1053/ j.jfas.2017.01.054.

6. Boulton AJM, Vileikyte L, Ragnarson-Tennvall G, Apelqvist J. The global burden of diabetic foot disease. Lancet 2005;366: 1719-24.

7. Misteli $H$, Kalbermatten $D$, Settelen C. Simple and complicated surgical wounds. Ther Umsch 2012;69:23-7. doi:10. 1024/0040-5930/a000246.

8. Baltzis D, Eleftheriadou I, Veves A. Pathogenesis and treatment of impaired wound healing in diabetes mellitus: new insights. Adv Ther 2014;31:817-36. doi:10.1007/s12325014-0140-x. Epub 2014 Jul 29.

9. Falanga V. Wound healing and its impairment in the diabetic foot. Lancet 2005;366:1736-43.

10. Oliver RA, Lovric V, Yu Y, et al. Development of a Novel Model for the Assessment of Dead-Space Management in Soft 
Leem $S$ et al.

Modified Lisfranc amputation

Tissue. PLoS One 2015;10:e0136514. doi:10.1371/journal. pone.0136514. eCollection 2015.

11. Agrawal A, Ayantunde AA, Cheung KL. Concepts of seroma formation and prevention in breast cancer surgery. ANZ J Surg 2006;76:1088-95.
12. Chen CF, Lin SF, Hung CF, et al. Risk of infection is associated more with drain duration than daily drainage volume in prosthesis-based breast reconstruction: a cohort study. Medicine (Baltimore). 2016;95:e5605. 